\title{
Incomplete Reporting of HIV/AIDS by Uganda's Surveillance System and the Associated Factors
}

\author{
Denis Akankunda Bwesigye ${ }^{1 *}$, Barry M. Loneck ${ }^{2}$, Barry R. Sherman ${ }^{3}$ \\ ${ }^{1}$ School of Public Health, Makerere University, Kampala, Uganda \\ ${ }^{2}$ School of Social Welfare, State University of New York, Albany, NY, USA \\ ${ }^{3}$ School of Public Health, State University of New York, Albany, NY, USA \\ Email: *akankunda.denis@gmail.com
}

Received 2 January 2016; accepted 18 April 2016; published 21 April 2016

Copyright (C) 2016 by authors and Scientific Research Publishing Inc.

This work is licensed under the Creative Commons Attribution International License (CC BY). http://creativecommons.org/licenses/by/4.0/

c) (i) Open Access

\section{Abstract}

Introduction: The United States government supported Ugandan government by introducing the District Health Information Software 2 (DHIS2) in 2012 to improve HIV/AIDS surveillance. Districts have yet to fully adopt this relatively new system given a $70.2 \%$ reporting completeness achieved nationally between April-June 2013. Methods: The study examined one dependent variable of districts' reporting completeness against four independent variables: 1) Number of client visits; 2) Number of district health units; 3) Number of NGOs delivering HIV/AIDS services; and 4) Regional location. The study employed cross-sectional study design which allowed researchers to compare many different variables at the same time. HIV/AIDS program data that were reported by districts into DHIS2 during the period of April to June 2013 were used to assess for reporting completeness. Findings: Districts with the lowest number of client visits (under 2500) achieved the highest mean reporting completeness $(81.6 \%)$, whereas a range of $2501-5000$, or over 5001client visits recorded $72.4 \%$ and $51.7 \%$ respectively. The higher the number of client visits is, the lower the reporting completeness is $(p<0.05)$. Those districts that were receiving support from only one and two NGO recorded $56.7 \%$ and $67.2 \%$ respectively. Districts supported by over three NGOs had the highest $(\mathbf{8 0 . 6 \% )}$ mean reporting completeness. NGOs-district support was statistically associated with reporting completeness $(p<0.05)$. The number of health units operated by a district was also significantly associated with reporting completeness $(p<0.05)$. The regional location of a district was not associated with reporting completeness $(p=0.674)$. Conclusion: The study results led us to recommend targeted future NGO support to districts with higher patient volume for HIV/AIDS services. Particularly, newly funded NGOs are to be established in districts operating over 40 health units. Incomplete reporting undermines identification of HIV-affected individuals and limits the ability to make evidence-based decisions regarding HIV/AIDS program planning and service delivery.

\footnotetext{
"Corresponding author.
}

How to cite this paper: Bwesigye, D.A., Loneck, B.M. and Sherman, B.R. (2016) Incomplete Reporting of HIV/AIDS by Uganda's Surveillance System and the Associated Factors. Open Journal of Preventive Medicine, 6, 125-132. 
Keywords

Incomplete Reporting, HIV/AIDS, Surveillance, Uganda

\section{Introduction}

Uganda has piloted a number of management information systems (MIS) to improve surveillance of HIV/AIDS during the last 20 years [1]. With support from the United States Agency for International Development (USAID), District Health Information Software (DHIS2) was introduced and scaled down to all districts in 2012. Introduction of DHIS2 nationally inducted districts into gradual transition from use of traditional paper medical records to electronic database. The ministry of health, with support from USAID funded HIV/AIDS projects, provides regular technical supervision to local districts to ensure equitable adaptation of the system. Enhanced surveillance of HIV/AIDS in Uganda has become increasingly important given a steady increase of HIV prevalence (6.4\% to $7.2 \%$ between 2009 and 2013) among the general population [2]. The HIV prevalence had dropped almost three-folds in the late 1990s, and long before such reductions were achieved elsewhere in the world. There is a major concern that new HIV infections have been steadily rising, e.g. from approximately 124,000 in 2009 to 128,000 in 2010 and to 145,000 by 2011. In fact, Uganda was in 2010 reported to have the fourth highest rate of HIV infections among fifty three African countries [3].

The data from sentinel surveillance sites recorded a decline in HIV prevalence from a high of $18 \%$ in the early 1990's to a low of approximately 5.2\% in 2004 which made Uganda a global icon in the fight against the epidemic. However, the national HIV prevalence in 2011 was reported to be $7.3 \%$ and even much higher (8.3\%) among women at [2]. A 2010 baseline of 102,157 new HIV cases was set for a new prevention plan dubbed 2011-2015 National HIV Prevention Strategy (NPS) that set out to reduce new cases to 71,510 by 2015 [4]. Surveillance for NPS targets was aided with the introduction of a District Health Management Information Software (DHIS2), a web-based database that collected and analysed routine HIV/AIDS program data in real-time. This initiative was launched in 2012 by the ministry of health with support from the US government. All local districts hospitals and private health care providers offering HIV/AIDS services were tasked to report their patient data through the DHIS2 to ensure equitable adaptation of the new technology.

Districts are responsible for entering all of the data into the DHIS2 as soon as they receive the medical forms from health facilities. Data reporting is carried out through a chain of ecological hierarchy of community health centers to hospitals, and then up to the district level. However, districts in Uganda have yet to fully adopt DHIS2 as revealed by low reporting completeness associated with medical records for outpatient, inpatient and antenatal services during the reporting period between April and June 2013. Researchers in the field of health systems have dubbed the process of "technological transfer" from developed to developing countries, as often carried out without taking into account of local contextual and cultural factors such as language, infrastructure, availability of personnel, appreciation of new health system technologies [5]-[9]. It is imperative that local districts adapt strongly to the use of DHIS 2 because achieving NSP target requires a functional national MIS that provides timely, accurate, and complete data on not only accurate numbers of new HIV infections, but also on com-prehensive HIV/AIDS programs like AIDS care and treatment.

\section{Methods}

We argue that investigating factors such as; number of HIV/AIDS clients served within a district in a particular reporting period; number of health faculties districts are required to report HIV/AIDS data on; number of US Government non-governmental organizations (NGOs) present and supporting a district to report HIV/AIDS program data; and regional location of districts, can provide insight to understanding how local districts are adapting to reporting of HIV/AIDS program data in DHIS2. The study adopted one dependent variable of reporting completeness attained by districts and measured as a continuous variable from zero $(0)$ to one hundred percent $(100 \%)$. We used cross-sectional study design which allows researchers to compare many different variables at the same time. HIV/AIDS program data reported during the period of April to June 2013 were used to assess for statistical analysis. We were aware of the weakness of cross-sectional study design, that they only offer a snapshot of a single moment in time; they do not consider what happens before or after that snapshot is 
taken. For instance, reporting completeness attained by districts nationally could have been far lower before or will be much higher after the study. Independent factors of: 1) Number of client visits recorded per district during the reporting period (April-June 2013); 2) Number of district health units; 3) Number of PEPFAR spell out NGOs delivering HIV/AIDS services; and 4) Regional location of districts, were assessed against the dependent variable. The independent factors, with exception of region, were subdivided into ordinal scales to assess for statistical differences in reporting completeness. Data analysis began with a simple examination of the bivariate relationships between each of the independent variables with the dependent variable. Hypotheses were tested using a factorial analysis of variance (ANOVA), followed by post-hoc t-tests using a Bonferronni correction. The operational definition of the study variables as well as the relevancy for their inclusion is explained as follows.

\subsection{Number of Client Visits}

Client visits refers to total number of individuals seeking HIV prevention and treatment services within a particular district during the months of April-June, 2013. Services range from voluntary counseling and testing (VCT), educational campaigns, antenatal services (ANC), early infant diagnosis of HIV, safe male circumcision (SMC) for HIV prevention, prevention of mother-to-child transmission of HIV (PMTCT), and anti-retroviral therapy (ART) for HIV positive individuals. To measure the impact of client load on reporting completeness among local districts, the number of client visits was grouped into ordinal of: 1) under 2500; 2) between 2501 and 5000; and 3) over 5001 .

\subsection{Number of NGOs Present in a District}

The United States Government's Presidential Emergency Plan for AIDS Relief (PEPFAR) is the major funder for the Uganda's HIV/AIDS programs. Between 2009 and 2010, the US government contributed 83\% and 93\% of the entire donor funding. Data on NGO support is only available on the US supported NGOs. These NGOs work in all local districts to implement HIV/AIDS programs. They equally support district health facilities to report their HIV/AIDS program data into the DHIS2. However Districts are disproportionally supported by PEPFAR NGOs (Implementing Partners-IPs), with some districts supported by over ten NGOs while others by only one or none. We hypothesize that reporting completeness is lower among those districts receiving less NGO support than others. Number of health units operated by the districts was also grouped into ordinal scales of: 1) under $20 ; 2$ ) between 21 and 40 ; and 3) over 41 .

\subsection{Number of Health Units}

Districts operate varying numbers of health units, some as few as ten while others over fifty. This disparity in numbers of health units operated by districts translated into a disparity in reporting workload faced by districts. This scenario is compounded by a shortage of health workers among districts. We hypothesize that districts operating far more health units than others are at a less disadvantage of reporting completeness of HIV/AIDS program data. Number of HIV/AIDS present in a district was equally grouped on an ordinal scale of: 1) only one; 2) only two; and 3) three and above.

\subsection{Region}

Uganda's health sector is managed through regional administrative structures of Central, Eastern, Northern and Western regions. Each one of these regions differs in terms of infrastructural development. Some regions e.g. the Central is has a well developed infrastructure like more stable and easily accessible internet, electricity, more health workers, etc, which could augment smooth adaptability of DHIS2 among districts located within this region. Districts located in the Northern and some parts of Eastern regions are more underdeveloped, primarily due to a 20 year civil war that ravaged this region. We hypothesize that a districts regional location will have an effect on reporting completeness of HIV/AIDS data in the DHIS. Districts' regional location of North, Central, Eastern and Western remained categorical variables.

\section{Results}

The factorial analysis of variance (Table 1) tested for the effects of client visits, number of NGOs, number of 
Table 1. Dependent variable: tests of between-subjects effects on reporting completeness.

\begin{tabular}{ccccccc}
\hline Source & Type III Sum of Squares & df & Mean Square & F & Sig. & Partial Eta Squared \\
\hline Corrected Model & $25,719.121^{\mathrm{a}}$ & 9 & 2857.680 & 17.117 & 0.000 & 0.639 \\
Intercept & $373,844.644$ & 1 & $373,844.644$ & $2.239 \mathrm{E} 3$ & 0.000 & 0.963 \\
Client visits & 5056.523 & 2 & 2528.261 & 15.144 & 0.000 & 0.258 \\
Number of NGOs & 4632.008 & 2 & 2316.004 & 13.872 & 0.000 & 0.242 \\
Number of health units & 3839.449 & 2 & 1919.724 & 11.499 & 0.000 & 0.209 \\
Region & 257.352 & 3 & 85.784 & 0.514 & 0.674 & 0.017 \\
Error & $14,524.728$ & 87 & 166.951 & & & \\
Total & $519,584.400$ & 97 & & & &
\end{tabular}

${ }^{\mathrm{a}} \mathrm{R}$ squared $=0.639$ (adjusted $\mathrm{R}$ squared $=0.602$ ).

health units and regions, on reporting completeness.

In the omnibus F-test, we obtained $\mathrm{F}=17.117(\mathrm{p}<0.05)$ and an effect size of 0.639 , indicating one or more of the independent variables has a significant association with mean reporting completeness. This warranted an examination of the F-statistics for each independent variable. The omnibus F-statistic $=17.117$ and $p<0.05$ and effect size of 0.639 .

\subsection{Client Visits}

There was a downward trend in mean reporting completeness as the number of client visits increased (Figure 1). Districts with the fewest client visits (under 2500) had the highest mean reporting completeness $(81.6 \%$ ); the scale 'between 2501 to 5000, and that of over 5001 attained reporting completeness of $72.4 \%$ and $51.7 \%$ respectively.

The mean differences in reporting completeness between groupings of number of client visits are reported in the multiple comparisons in Table 2 above. The first two rows represent groupings of "under 2500" to "between 2501 - 5000", and "over 5001" client visits. The results indicate that client visits has a significant effect on reporting completeness with $\mathrm{F}(15.144)$ and $\mathrm{p}<0.05$.

\subsection{Number of NGOs}

There was an upward trend in mean reporting completeness as the number of NGOs increased (see Figure 2). Districts that were receiving support from only one compared to those supported by two NGOs attained $56.7 \%$ and $67.2 \%$ mean reporting completeness respectively. The districts receiving supported by over three NGOs had the highest $(80.6 \%)$ mean reporting completeness.

The number of NGOs also had a significant effect on reporting completeness with $\mathrm{F}(13.872)$ and $\mathrm{p}<0.05$. Moreover the mean reporting completeness attained by "only one NGO" was 23.912 lower than that of "three+ NGOs" and the difference was statistically significant $(\mathrm{p}<0.05)$ (Table 3$)$.

\subsection{Number of Health Units}

There was a downward trend in mean reporting completeness as the number of health units increased (Figure 3 ). Districts with fewest ( $<20$ ' health units) had the highest mean reporting completeness $(84.5 \%)$. We observed that an increase in a scale for health units from, between 21 and 40, and further to over 41 led to steady reduction in mean reporting completeness from $74.6 \%$ to $54.3 \%$. Notably, districts operating over 41 health units attained lowest mean reporting completeness (54.3\%).

The mean differences between groupings of the health units are reported in the multiple comparisons above. 


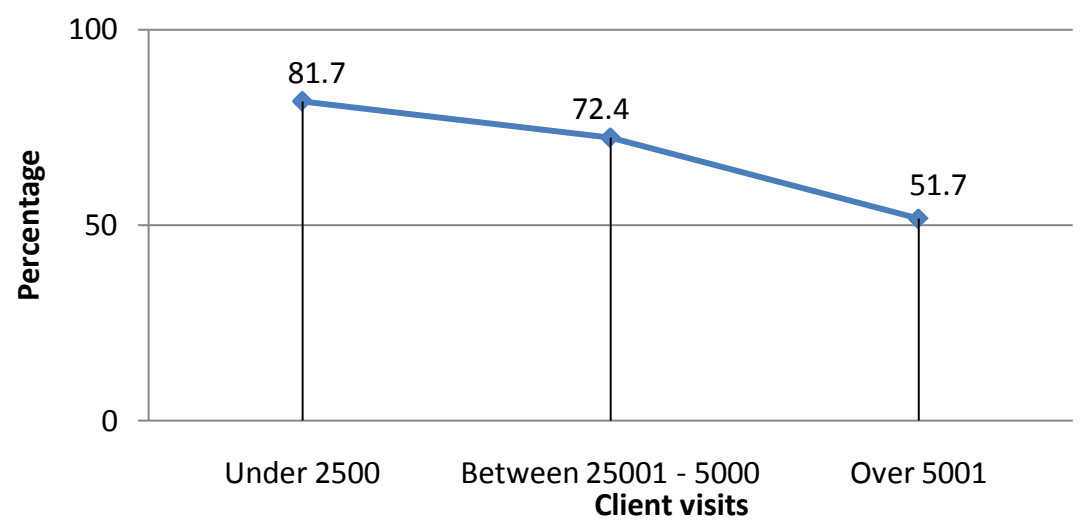

Figure 1. Mean reporting completeness by client visits.

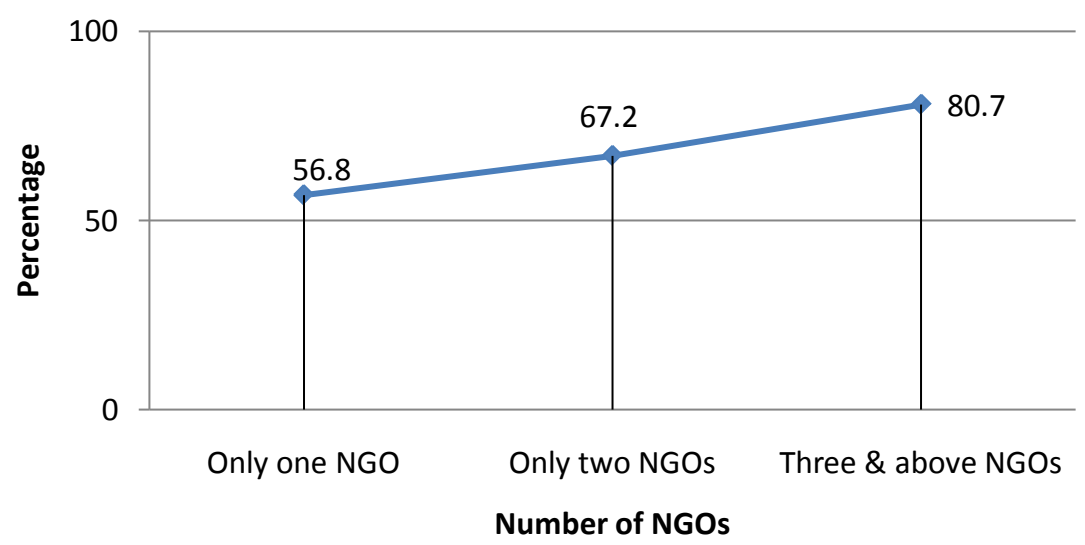

Figure 2. Mean reporting completeness by the number of NGOs.

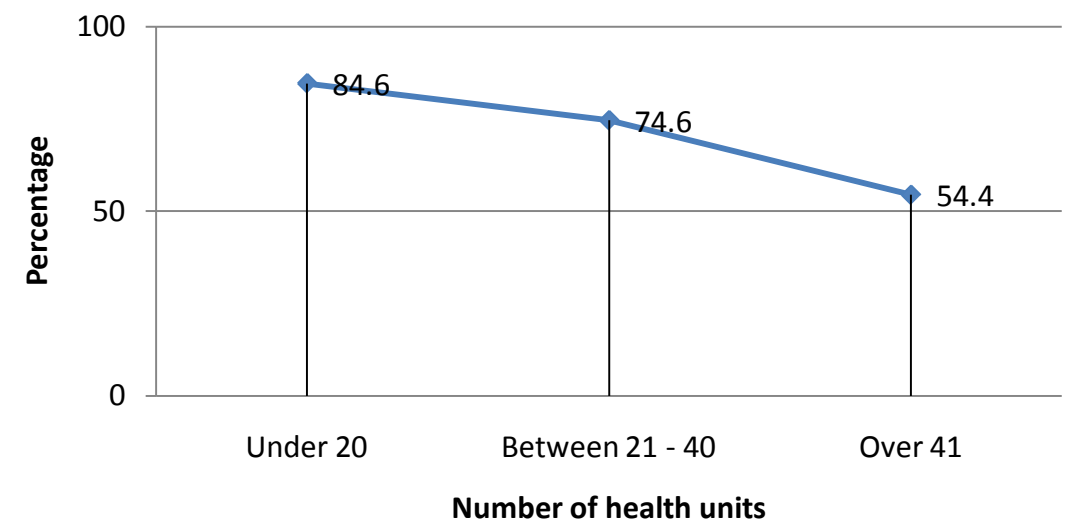

Figure 3. Mean reporting completeness by the number of health units.

The first two rows represent groupings of "under 20" to "between 21 - 40", and "over 41" health units. The mean reporting completeness for the "under 20" health units was 30.216 higher than the "over 41" health units and the difference was statistically significant $(\mathrm{p}<0.05)$ (Table 4$)$.

\subsection{Regions}

Across regions, mean reporting completeness appeared to be approximately equal (see Figure 4). Central72.4\%; Eastern-70.6\%; Northern-68.6\%; and Western-69.5\%. District's regional location did not have a significant effect on reporting with $\mathrm{F}(0.514)$ and $\mathrm{p}=0.674$. 
Table 2. Multiple comparisons for client visits on reporting completeness/bonferroni.

\begin{tabular}{ccccccc}
\hline $\begin{array}{c}\text { (I) Number of client } \\
\text { visits }\end{array}$ & (J) Number of client visits & $\begin{array}{c}\text { Mean Difference } \\
\text { (I-J) }\end{array}$ & Std. Error & Sig. & \multicolumn{2}{c}{$95 \%$ Confidence Interval } \\
\cline { 5 - 6 } Under 2500 & Between 2501 - 5000 & $9.253^{*}$ & 3.3614 & 0.022 & 1.048 & 17.459 \\
\multirow{2}{*}{ Between 2501 - 5000 } & Over 5001+ & $29.933^{*}$ & 3.0455 & 0.000 & 22.499 & 37.368 \\
& Under 2500 & $-9.253^{*}$ & 3.3614 & 0.022 & -17.459 & -1.048 \\
\multirow{2}{*}{ Over 5001+ } & Over 5001+ & $20.680^{*}$ & 3.6268 & 0.000 & 11.826 & 29.534 \\
& Under 2500 & $-29.933^{*}$ & 3.0455 & 0.000 & -37.368 & -22.499 \\
\hline
\end{tabular}


the null which assumes equal means.

Table 3. Multiple Comparisons for NGOs on reporting completeness/ Bonferroni.

\begin{tabular}{|c|c|c|c|c|c|c|}
\hline \multirow{2}{*}{ (I) Number of NGOs } & \multirow{2}{*}{ (J) Number of NGOs } & \multirow{2}{*}{$\begin{array}{l}\text { Mean Difference } \\
(\mathrm{I}-\mathrm{J})\end{array}$} & \multirow{2}{*}{ Std. Error } & \multirow{2}{*}{ Sig. } & \multicolumn{2}{|c|}{$95 \%$ Confidence Interval } \\
\hline & & & & & Lower Bound & Upper Bound \\
\hline \multirow{2}{*}{ Only one NGO } & Only two NGOs & $-10.459^{*}$ & 3.5166 & 0.011 & -19.044 & -1.875 \\
\hline & Three+ & $-23.912^{*}$ & 3.1727 & 0.000 & -31.657 & -16.167 \\
\hline \multirow{2}{*}{ Only two NGOs } & Only one NGO & $10.459^{*}$ & 3.5166 & 0.011 & 1.875 & 19.044 \\
\hline & Three + & $-13.453^{*}$ & 3.1727 & 0.000 & -21.198 & -5.708 \\
\hline \multirow{2}{*}{ Three+ } & Only one NGO & $23.912^{*}$ & 3.1727 & 0.000 & 16.167 & 31.657 \\
\hline & Only two NGOs & $13.453^{*}$ & 3.1727 & 0.000 & 5.708 & 21.198 \\
\hline
\end{tabular}

Based on observed means. The error term is Mean Square (Error) $=166.951 .^{*}$ The mean difference is significant at the 0.05 level therefore we reject the null which assumes equal means.

Table 4. Multiple comparisons for health units on reporting completeness/bonferroni.

\begin{tabular}{|c|c|c|c|c|c|c|}
\hline \multirow{2}{*}{ (I) Health units } & \multirow{2}{*}{ (J) Health units } & \multirow{2}{*}{ Mean Difference (I-J) } & \multirow{2}{*}{ Std. Error } & \multirow{2}{*}{ Sig. } & \multicolumn{2}{|c|}{$95 \%$ Confidence Interval } \\
\hline & & & & & Lower Bound & Upper Bound \\
\hline \multirow{2}{*}{ Under 20} & Between $21-40$ & $9.957^{*}$ & 3.3104 & 0.010 & 1.876 & 18.038 \\
\hline & Over 41 & $30.216^{*}$ & 3.4260 & 0.000 & 21.853 & 38.580 \\
\hline \multirow{2}{*}{ Between $21-40$} & Under 20 & $-9.957^{*}$ & 3.3104 & 0.010 & -18.038 & -1.876 \\
\hline & Over 41 & $20.259^{*}$ & 3.0561 & 0.000 & 12.799 & 27.720 \\
\hline \multicolumn{7}{|c|}{ Continued } \\
\hline \multirow{2}{*}{ Over 41 health units } & Under 20 & $-30.216^{*}$ & 3.4260 & 0.000 & -38.580 & -21.853 \\
\hline & Between $21-40$ & $-20.259^{*}$ & 3.0561 & 0.000 & -27.720 & -12.799 \\
\hline
\end{tabular}

Based on observed means. The error term is Mean Square (Error) $=166.951 .{ }^{*}$ The mean difference is significant at the 0.05 level therefore we reject the null which assumes equal means.

\section{Discussion}

We found that an increase in client visits was associated with a reduction in mean reporting completeness attained in DHIS2 by districts. This finding was consistent with a related study in South Africa, which noted that performance of health information systems was poor especially among hospitals that served high volumes of patients [10]. According to the 2011 Human Resources for Health Audit report, Uganda has a health worker staffing shortage of $42 \%$. This staffing situation might be much worse among districts categorized as hard-to-reach. Uganda's hospitals face severe health worker shortages with a low doctor-patient ratio of 1 to 24,725 , nurse/ 


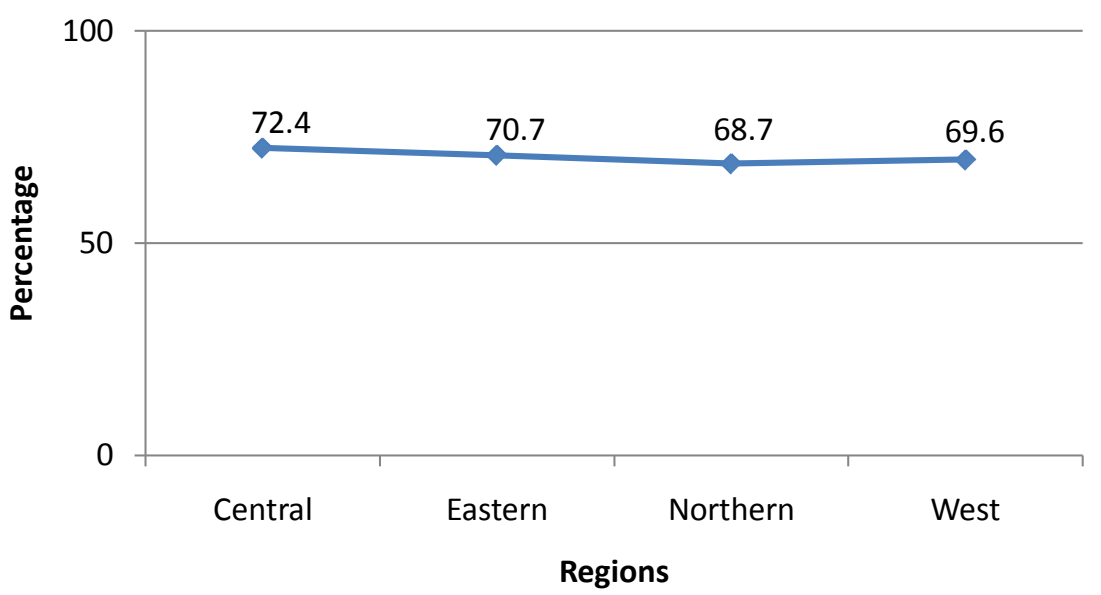

Figure 4. Mean reporting completeness by the regions.

midwife to patient ratio of $1: 11,000$ [11].

The shortage of health workers compounded by high volumes of patients undermine the already little capacity of districts to attain high reporting completeness of HIV/AIDS data into the government's DHIS2. Regarding NGO support to districts' HIV/AIDS programming, districts are disproportionally supported by PEPFARfunded NGOs. We hypothesized that reporting completeness would be lower among districts receiving support from fewer NGO. Indeed we established that the number of HIV/AIDS NGOs present in a district had positive impact of reporting completeness attained in DHIS2 and this relationship was found to be statistically significant at $F(13.872)$ and $p<0.05$. We found a negative impact on the number of health units operated by district and the associated reporting completeness. The statistical analysis observed that a consistent increase in the number of health units operated by districts was associated with a consistent decrease in mean reporting completeness.

To estimate HIV prevalence among the general population, Uganda relies primarily on data from HIV tests of pregnant women seeking antenatal services. However, reporting completeness of medical records for antenatal services was a mere $37 \%$ between April and June 2013. Moreover, there was a drop from $24 \%$ to $34 \%$ between 2010 and 2011 in the HIV exposed babies, whose mothers tested HIV positive and were enrolled on antiretroviral drugs. Although Uganda's health sector has been making steady progress in rolling out an electronic medical records management, an overall $70.3 \%$ HIV/AIDS reporting completeness attained by districts nationally represents a major gap in the countries DHIS2.

\section{Conclusion}

Although data for this study were extracted from a short reporting period (April-June 2013), the sample was nationally representative. Therefore these study findings can be leveraged to inform evidence-based policies for improved HIV/AIDS surveillance at all district levels. Ultimately, the reporting completeness will have to be elevated to $100 \%$ in all 132 Uganda's districts. To be noteworthy is that allocation of PEPFAR implementing partners (IPs) does not follow any standard criteria for apportioning NGO support among local districts. Some important findings from this study, e.g. the associated role of client visits, number of HIV/AIDS NGOs present in a district, number of health units operated by districts, should be targeted with actions plans targeting those most disadvantaged districts.

\section{Authors' Contribution}

DBA conceived of the study, and participated in its design and helped to draft the manuscript. BRS participated in the conceptual development of the study and coordinated the team. BML developed the statistical analysis plan for the study and participated in data analysis, interpretation and presentation. All authors read and approved the final manuscript.

\section{References}

[1] Bongguk, J., Jesse, T., Virga, P. and Sergey, V. (2012) Electronic Health Information Technology as a Tool for Im- 
proving Quality of Care and Health Outcomes for HIV/AIDS Patients. International Journal of Medical Informatics, 81, e39-e45.

[2] Uganda AIDS Commissions (2012) The Global AIDS Progress Report. Uganda Country Report.

[3] The Joint United Nations Programme on AIDS (2012) Global AIDS Response Progress Report. Country Progress Report, Uganda.

http://www.unaids.org/en/dataanalysis/knowyourresponse/countryprogressreports/2012countries/ce_UG_Narrative_Re port[1].pdf

[4] Ministry of Health (2011) Human Resources for Health Biannual Report. Uganda.

http://library.health.go.ug/publications/health-workforce/human-resource-management/human-resources-health-audit-r eport-2010

[5] Nhampossa, J.L. (2005) Re-Thinking Technology Transfer as Technology Translation: A Case Study of Health Information Systems in Mozambique. Faculty of Mathematics and Natural Sciences, University of Oslo, Oslo. http://heim.ifi.uio.no/ jensj/Nhampossa2005TechTransferTechTranslationHIS.pdf

[6] Robey, D., Boudreau, M.C. and Rose, G.M. (2000) Information Technology and Organizational Learning: A Review and Assessment of Research. Accounting, Management and Information Technologies, 10, 125-155. http://dx.doi.org/10.1016/S0959-8022(99)00017-X

[7] Orlikowski, W.J. and Robey, D. (1991) Information Technology and the Structuring of Organizations. Journal of Information Systems Research, 2, 143-169. http://dx.doi.org/10.1287/isre.2.2.143

[8] Bødker, S. (1998) Understanding Representation in Design. Human Computer Interaction, 13, 107-125. http://dx.doi.org/10.1207/s15327051hci1302 1

[9] Garrety, K. and Badham, R. (2000) The Politics of Sociotechnical Intervention: An Interactionist View. Technology Analysis \& Strategic Management, 12, 103-118. http://dx.doi.org/10.1080/095373200107265

[10] Coovadia, H., Jewkes, R., Barron, P., Sanders, D. and McIntyre, D. (2009) The Health and Health System of South Africa: Historical Roots of Current Public Health Challenges. The Lancet, 374, 817-834. http://dx.doi.org/10.1016/S0140-6736(09)60951-X

[11] (2011) Uganda Bureau of Statistics (UBOS) and Macro International Inc. Uganda AIDS Indicator Survey 2011. 